\title{
Molecular mechanism of protrusion formation during cell-to-cell spread of Listeria
}

\author{
Keith Ireton ${ }^{1 *}$, Luciano A. Rigano ${ }^{1}$, Lilia Polle $^{2}$ and Wolf-Dieter Schubert ${ }^{3 *}$ \\ 1 Department of Microbiology and Immunology, University of Otago, Dunedin, New Zealand \\ 2 Department of Biotechnology, University of the Western Cape, Bellville, Cape Town, South Africa \\ ${ }^{3}$ Department of Biochemistry, University of Pretoria, Pretoria, South Africa
}

\section{Edited by:}

Stephanie M. Seveau, The Ohio

State University, USA

Reviewed by:

Mikhail A. Gavrilin, Ohio State

University, USA

Hélène Marquis, Cornell University, USA

Guy T. V. Nhieu, Collège de France,

France

\section{${ }^{*}$ Correspondence:}

Keith Ireton, Department of

Microbiology and Immunology,

University of Otago,

720 Cumberland Street, PO Box 56,

Dunedin 9054, New Zealand

e-mail: keith.ireton@otago.ac.nz;

Wolf-Dieter Schubert, Department of Biochemistry, University of

Pretoria, Private Bag X20, Hatfield,

Pretoria 0028, South Africa

e-mail:wolf-dieter.schubert@

up.ac.za

\section{INTRODUCTION}

Several bacteria, including the enteric pathogens Listeria monocytogenes and Shigella flexneri and select species of the arthopodborne genus Rickettsia, use an actin-based motility process to actively spread within human tissues (Gouin et al., 2005; Haglund and Welch, 2011; Ireton, 2013). After internalization into human cells, bacteria induce the lysis of host-derived membrane vacuoles, and subsequently replicate in the cytoplasm (Figure 1A; steps 1-3). Cytosolic bacteria stimulate the assembly of actin filaments, resulting in intracellular motility (step 4). Listeria and Shigella have mechanisms to evade autophagy- a host degradative pathway that would other otherwise kill cytoplasmic microbes (Cemma and Brumell, 2012; Mostowy and Cossart, 2012). Bacteria propelled by actin-based motility ultimately contact and remodel the host plasma membrane into thin membrane projections termed "protrusions" (Figure 1A, step 5). These protrusions are internalized by neighboring cells, resulting in microbes in double-membranous vacuoles (step 6). Finally, the vacuoles are destroyed by bacterial factors, liberating bacteria into the cytosol of the newly infected cell.

Much progress has been made in understanding the mechanisms of F-actin assembly by Listeria, Shigella, and Rickettsia (Gouin et al., 2005; Haglund and Welch, 2011; Ireton, 2013). In brief, the Listeria surface protein ActA and Shigella protein IcsA each activate a mammalian actin polymerization machinery called the Arp $2 / 3$ complex. ActA is a direct activator ("nucleation promoting factor") of Arp2/3, whereas IcsA stimulates Arp2/3 through recruitment of a human nucleation promoting factor known as N-WASP. Unlike ActA or IcsA, the Rickettsia surface protein Sca2 directly promotes assembly of actin filaments independently of Arp2/3 or other host factors. Sca2 may act as a functional mimic of a class of eukaryotic actin polymerization proteins called formins. Compared to the fairly detailed understanding of F-actin assembly by Listeria, Shigella, and Rickettsia, much less is know about how these pathogens produce protrusions that mediate cell-to-cell spread. This mini-review focuses on recent studies with Listeria that have shed light on the molecular mechanism of protrusion formation.

\section{Listeria PROTRUSIONS ARE CONTROLLED BY THE BACTERIAL PROTEIN InIC AND ITS HOST LIGAND Tuba}

Recent work led to the identification of microbial and human proteins that regulate protrusion formation by Listeria (Rajabian et al., 2009). A bacterial mutant deleted for the gene inlC $(\Delta \mathrm{inlC})$ was found to be partly defective in in cell-to-cell spread in the polarized human cell line Caco-2 BBE1. Confocal microscopy analysis indicated that the $\triangle i n l C$ mutant is unaffected in phagosomal escape or in the ability to produce F-actin tails. Instead the mutant is compromised in the formation of protrusions, producing these structures at only about $50 \%$ the efficiency of the wild-type bacterial strain.

InlC is a member of the internalin family of Listeria proteins (Engelbrecht et al., 1996). These proteins share a common 
A

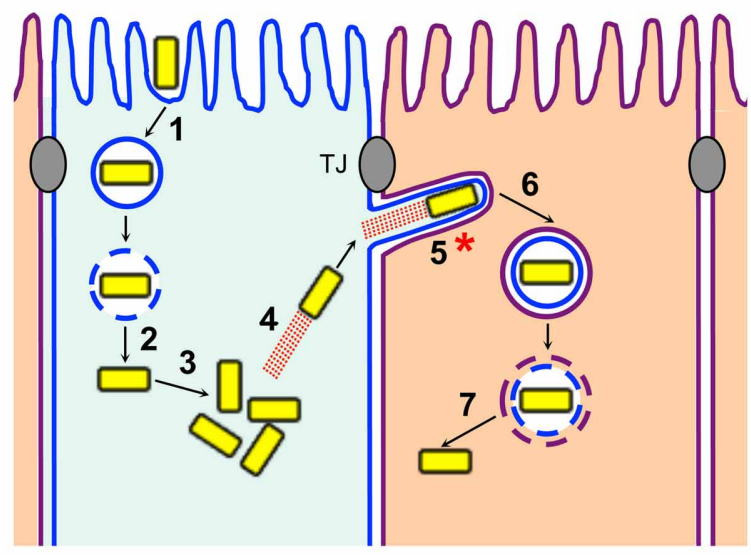

C
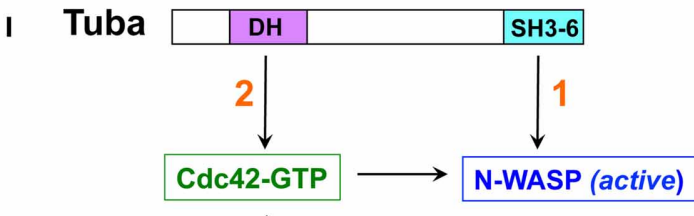

?

Inhibition of Listeria

protrusion formation
B

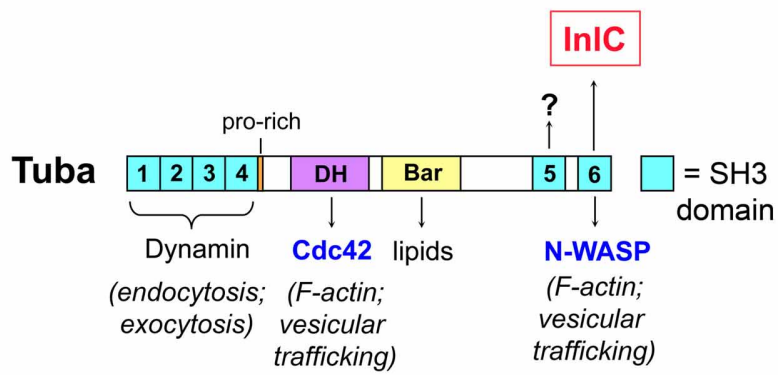

II Tuba

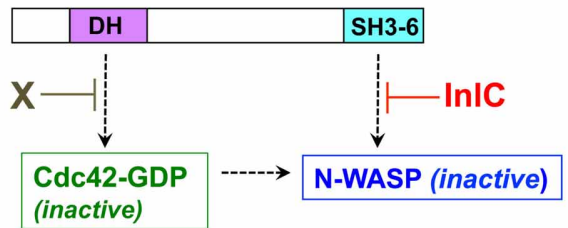

Listeria protrusion formation
D

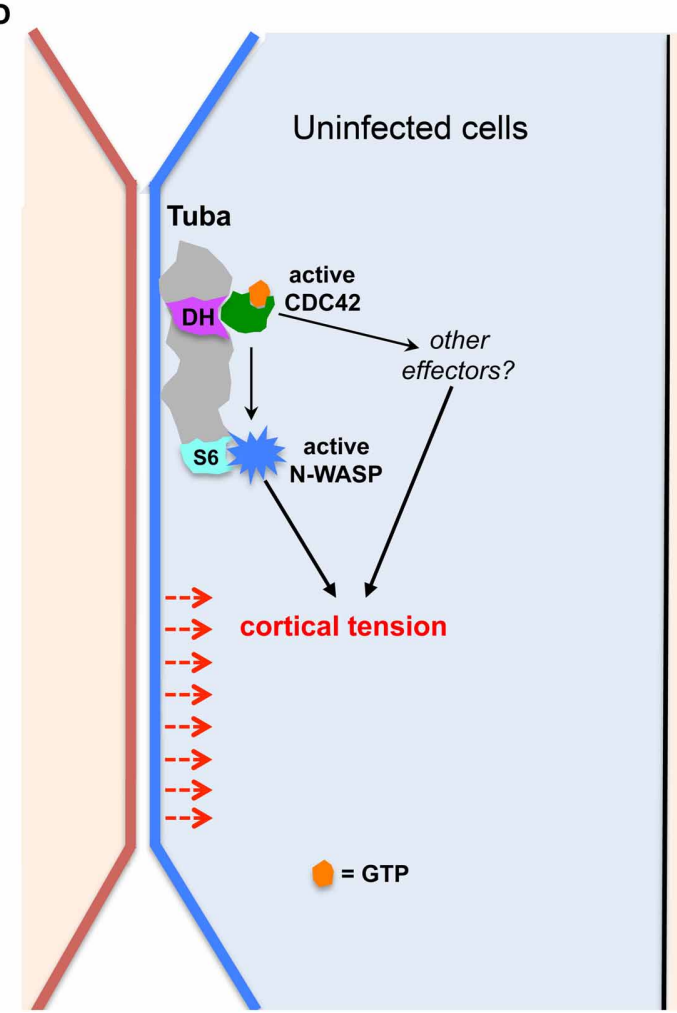

FIGURE 1 | Bacterial and host factors controlling protrusion formation in polarized epithelial cells. (A) Steps in the intracellular life cycle of Listeria. (1) internalization of bacteria into host cells, (2) escape from phagosomes, (3) bacterial replication in the cytosol, (4) actin based motility, (5) formation of bacterial protrusions, (6) engulfment of protrusions, and (7) dissolution of the

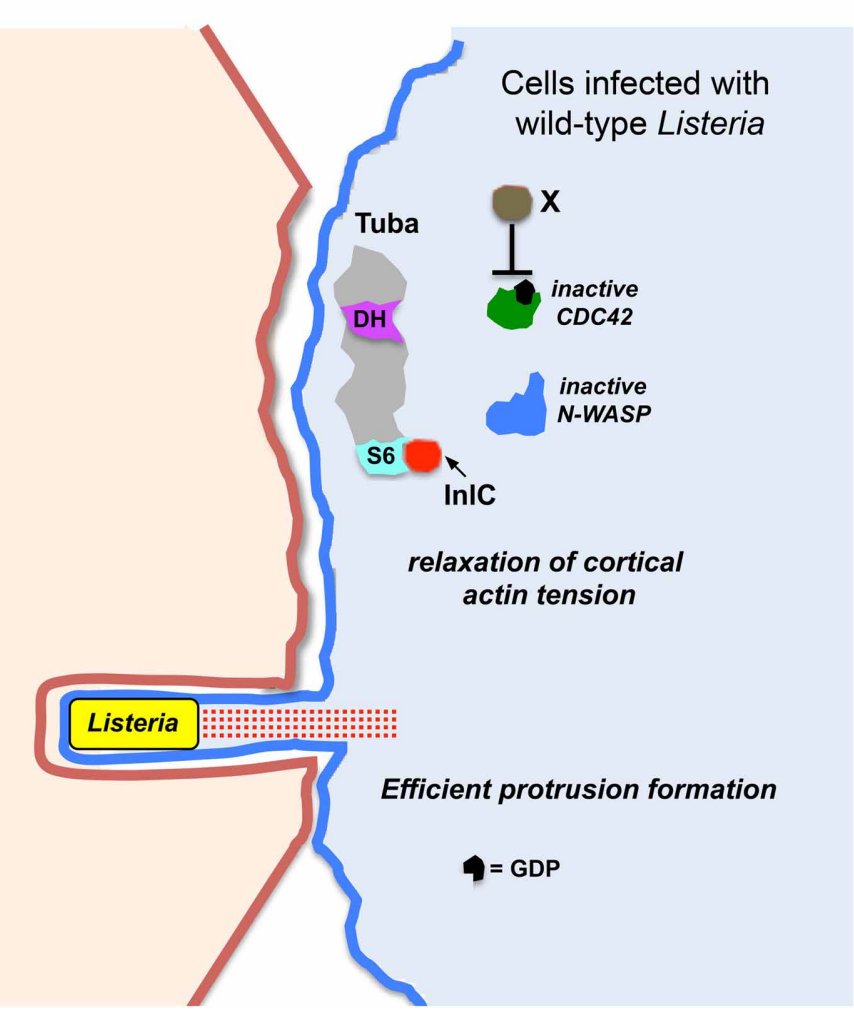

double membranous vacuole. The process of cell-cell spread comprises steps 4-7. This minireview focuses on molecular events controlling protrusion formation (indicated with an asterisk). "TJ" denotes tight junctions. (B) Domain structure of the human scaffolding protein Tuba. SH3 domains

(Continued) 


\section{FIGURE 1 | Continued}

(light blue) are numbered 1-6. SH3 domains 1-4 bind the GTPase Dynamin, whereas $\mathrm{SH} 3$ domain 6 (SH3-6) interacts with N-WASP. SH3-6 is also targeted by the secreted listerial protein InIC. Ligands of SH3-5 are unknown. The Dbl Homology (DH) domain has guanine nucleotide exchange activity for the human GTPase Cdc42. The Bar domain presumably binds lipids of the plasma membrane inner leaflet. Physiological processes controlled by Tuba ligands are listed. (C) Relief of Tuba-mediated inhibition of Listeria spreading by InIC and other bacterial factors: (i) Without InIC or other bacterial proteins, listerial protrusion formation is restrained by [1] Tuba SH3-6 binding to $\mathrm{N}$-WASP to recruit N-WASP to the plasma membrane and/or activate N-WASP, and [2] Tuba-mediated stimulation of Cdc42 to impair protrusions by activating N-WASP and/or other effectors (?). (ii). InIC relieves Tuba-mediated inhibition of bacterial spread by blocking N-WASP binding to Tuba SH3-6. In addition, an unknown bacterial factor " $\mathrm{X}$ " promotes protrusion formation by reducing Cdc42 activity. (D) Model of tight junction perturbation by $\operatorname{InIC}$. In uninfected polarized epithelial cells or cells infected with $\triangle$ in/C bacteria (left panel), host Tuba, N-WASP and Cdc42 together promote cortical tension presumably via Tuba-mediated activation of $\mathrm{Cdc} 42$ and of N-WASP as well as Cdc42-GTP activation of N-WASP and possibly of additional effectors. In cells infected with wild-type Listeria (right panel), cortical tension is diminished by InIC displacing N-WASP from Tuba SH3-6 and by Cdc42 inhibition via an unidentified bacterial factor " $\mathrm{X}$." Reduced tension allows efficient protrusion formation by Listeria. architecture including an amino terminal cap domain, a leucinerich-repeat (LRR) domain that often mediates interaction with host proteins and a immunoglobulin-like interrepeat domain (Schubert et al., 2001; Bierne et al., 2007). Interestingly, although most internalin proteins are anchored to the Listeria cell surface, InlC is entirely secreted (Engelbrecht et al., 1996). Importantly, the expression of inlC is greatly increased upon internalization of bacteria into human cells due to the action of the bacterial transcription factor PrfA (Engelbrecht et al., 1996; Rajabian et al., 2009; Gouin et al., 2010). The intracellular expression of InlC and presence of an LRR domain in this protein suggested that InlC might stimulate Listeria protrusions by interacting with a cytoplasmic host target. Such a target, the human scaffolding protein Tuba, was identified through a yeast two-hybrid screen of human cDNAs using InlC as bait (Rajabian et al., 2009).

Tuba is a $177 \mathrm{kDa}$ protein scaffolding protein involved in F-actin assembly (Salazar et al., 2003; Kovacs et al., 2006; Otani et al., 2006), cell junction regulation (Otani et al., 2006), cell morphogenesis (Bryant et al., 2010; Qin et al., 2010; Kovacs et al., 2011), and exocytosis (Bryant et al., 2010; Sato et al., 2012). Tuba comprises several functional domains, including a potentially lipid binding Bar domain, a Dbl homology (DH) domain that activates the GTPase Cdc42, and six Src Homology 3 (SH3) domains (Salazar et al., 2003; Otani et al., 2006) (Figure 1B). The first four SH3 domains in Tuba interact with the GTPase Dynamin, whereas the last SH3 domain (termed "SH3-6") binds several human ligands, including the actin regulatory protein N-WASP (Salazar et al., 2003). Ligands of the fifth SH3 domain remain to be identified. Importantly, InlC binds to Tuba SH3-6, displacing host N-WASP (Rajabian et al., 2009).

Experiments with polarized Caco-2 BBE1 cells indicate that Tuba limits protrusion formation of $\triangle i n l C$ mutant Listeria (Rajabian et al., 2009). Specifically, the defect in protrusions normally observed with this mutant is suppressed when Tuba is depleted through RNA interference (RNAi). Importantly, Tuba depletion has no effect on protrusion formation by wild-type Listeria expressing InlC. These genetic data indicate that host Tuba has the potential to impair Listeria spread unless bacteria intervene by producing InlC, which antagonizes Tuba. Biochemical and genetic studies indicate that one of the ways that InlC inhibits Tuba is by binding to its SH3-6 domain, thereby disrupting Tuba/N-WASP complexes. Thus, Tuba and N-WASP comprise a host signaling pathway that must be antagonized by InlC in order for Listeria to spread efficiently (Figure 1C).

\section{ROLE OF HOST Cdc42 IN CONTROLLING Listeria PROTRUSIONS}

The presence of multiple protein or lipid binding domains in Tuba (Figure 1B) prompts the question as to whether Tuba ligands apart from N-WASP affect Listeria spread. Interestingly, recent data indicates that the Tuba effector Cdc42 controls bacterial protrusions.

Cdc42 is a human GTPase that regulates many biological processes, including cell motility, endocytic and exocytic trafficking of vesicles, the formation and maintenance of cell junctions, and cell polarity (Jaffe and Hall, 2005; Otani et al., 2006; Harris and Tepass, 2010). The DH domain of Tuba activates Cdc42, without affecting related GTPases (Salazar et al., 2003; Otani et al., 2006). Importantly, Listeria actively antagonizes host Cdc42 in order to promote bacterial spread (Rigano et al., 2014). Infection of Caco2 BBE1 cells causes a $\sim 65 \%$ reduction in levels of Cdc42-GTP. In addition, a dominant negative allele of $\mathrm{Cdc} 42$ restores normal protrusion formation to $\triangle$ inlC mutant Listeria, whereas constitutively activated Cdc42 inhibits protrusions normally made by wild-type bacteria. These latter findings indicate that the ability to inactivate $\mathrm{Cdc} 42$ is required for efficient bacterial protrusion formation. Interestingly, the effect of Listeria on Cdc42 is largely independent of InlC, and is instead due to an unidentified bacterial factor. The biochemical mechanism by which this factor antagonizes the host GTPase is presently unknown.

A model for how Tuba, N-WASP, and Cdc42 act together to regulate bacterial spread is presented in Figure 1C. In the absence of InlC, Tuba restrains Listeria protrusion formation by using its SH3-6 domain to engage N-WASP and its $\mathrm{DH}$ domain to activate Cdc42. Given the ability of Cdc42-GTP to activate N-WASP (Suetsugu and Gautreau, 2012), this GTPase may limit protrusions by acting solely through N-WASP. In such a scenario, simultaneous regulation by $\mathrm{SH} 3-6$ and $\mathrm{Cdc} 42$ would stimulate N-WASP activity in an additive fashion (Carlier et al., 2000; Suetsugu and Gautreau, 2012). Another possibility is that Cdc42-GTP controls bacterial protrusions through a host effector apart from N-WASP. To date, more than 30 effector proteins are known to interact with the GTP bound form of mammalian Cdc42 (Wallace et al., 2010). Future work may uncover functions for some of these effectors in limiting bacterial spread. Wildtype Listeria relieves Tuba-mediated inhibition in protrusions by using InlC to block interaction of the Tuba SH3-6 domain with N-WASP (Figure 1C). In addition, bacteria produce an unidentified factor ("X") that antagonizes host Cdc42. Through these 
effects on N-WASP and Cdc42, Listeria overcomes the barrier to spread that would otherwise by imposed by the Tuba signaling pathway.

\section{InIC PERTURBS HOST CELL JUNCTIONS}

Tuba, N-WASP, and Cdc42 each control the structure of cellcell junctions in epithelial cells. Specifically, depletion of Tuba or N-WASP by RNAi or dominant negative inhibition of Cdc42 causes normally linear tight junctions to slacken (Otani et al., 2006; Rajabian et al., 2009). It is thought that these curved junctions reflect a role for Tuba, N-WASP, and Cdc42 in producing cortical tension at the plasma membrane. Importantly, infection of Caco-2 BBE1 cells with wild-type Listeria alters tight junctions similarly to inhibition of Tuba, N-WASP, or Cdc42 (Rajabian et al., 2009). By contrast, infection with $\triangle$ inlC mutant bacteria fails to affect junctions. Ectopic expression of InlC in the absence of bacteria is sufficient to induce curved junctions. Finally, expression of a constitutively activated allele of Cdc42 restores normal linear junctions to cells infected with wild-type Listeria (Rigano et al., 2014). Collectively, these results indicate that InlC perturbs cell junctions through inhibition of Tuba, N-WASP, and Cdc42. By slackening junctions, InlC likely diminishes cortical tension at the host plasma membrane. Such decreased tension may augment Listeria spread by removing an inward force that would otherwise oppose bacterial protrusions. Host Tuba, N-WASP, and Cdc42 can be therefore viewed as a junctional regulatory system that has the potential to restrict bacterial spread. By using InlC to antagonize this regulatory system, Listeria circumvents host restriction of spreading (Figure 1D).

\section{InIC-Tuba INTERACTION AFFECTS VIRULENCE}

Genetic data indicate that interaction of InlC with Tuba is needed for efficient cell-to-cell spread in cultured human cells. An alanine substitution of lysine 173 in InlC (K173A) results in a protein that folds normally, but is partly defective in binding the Tuba SH3-6 domain (Rajabian et al., 2009). Importantly, a Listeria inlC.K173A strain expressing the mutant InlC protein is compromised for protrusion formation in polarized Caco-2 BBE1 cells. In addition to interacting with Tuba, InlC binds the host protein IKK $\alpha$ (Gouin et al., 2010). InlC-IKK $\alpha$ interaction inhibits the $\mathrm{NF} \mathrm{KB}$ signaling pathway, thereby dampening production of host pro-inflammatory cytokines. The K173A mutation in InlC does not effect Listeria-mediated inhibition of $\mathrm{NF} \mathrm{B}$, indicating that this mutation specifically uncouples InlC from host Tuba (Leung et al., 2013). Consequently, studies with the Listeria inlC.K173A strain indicate that efficient cell-cell spread in cultured cells requires the ability of InlC to bind Tuba. By contrast, regulation of NFKB by InlC likely does not affect bacterial spread.

A recent study took advantage of the inlC.K173A strain to address if InlC-Tuba interaction is needed for virulence in a mouse model (Leung et al., 2013). In intravenously inoculated animals, the inlC.K173A mutant strain had a 50\% lethal dose $\left(\mathrm{LD}_{50}\right)$ that was about 5 -fold higher than that of the isogenic wild-type strain. Importantly, this increase in $\mathrm{LD}_{50}$ was essentially identical to that observed for a mutant strain deleted for the inlC gene $(\Delta i n l C)$. Compared to the wild-type strain, the inlC.K173A mutant strain exhibited lower bacterial loads in the liver. Histological analysis of livers indicated that the inlC.K173A strain produced smaller foci of infection than did the wild-type strain. These smaller foci are consistent with a role for InlC in cell-cell spread in vivo. Taken together, these results provide evidence that interaction of InlC with host Tuba is important for full virulence.

\section{STRUCTURAL ELUCIDATION OF Tuba INHIBITION BY InIC}

The molecular and atomic details underlying the displacement of N-WASP from Tuba SH3-6 by InlC (Rajabian et al., 2009) were recently investigated structurally and biophysically (Polle et al., 2014) (Figure 2).

The structural analysis of Tuba SH3-6 with its physiological partner N-WASP, revealed a typical SH3 domain structure for Tuba SH3-6 consisting of a five-stranded, antiparallel $\beta$-barrel with a 310 -helix in loop $\beta 4-\beta 5$ (Polle et al., 2014). The prolinerich peptide ${ }^{346}$ pppalpssapsg ${ }^{357}$ of N-WASP adopts a polyproline type II (PPII) helical conformation and binds in a class II orientation (Lim et al., 1994) to the peptide binding loop of Tuba SH3-6 located between the conserved loops known as RT-Src $(\beta 1-\beta 2$ loop) and $n-S r c(\beta 3-\beta 4)$ (Figure 2$)$. The surface area of Tuba SH3-6 and of the peptide involved in the interaction, respectively, amount to $\sim 500$ and $600 \AA^{2}$ or 10 and $44 \%$ of the respective total. Despite this substantial interaction a dissociation constant of $46.5 \pm 5 \mu \mathrm{M}$ as determined by isothermal titration calorimetry implies a weak and hence transitory interaction. Interestingly, biophysical and crystallographic experiments indicate that the Tuba SH3-6 domain additionally interacts with other Tuba SH3-6 monomers through asymmetric contacts involving two additional parts of its surface distinct from the N-WASP peptide binding groove. Tuba may thus, form higher order structures via this SH3-6 domain (Polle et al., 2014).

A co-crystal structure of Tuba SH3-6 in complex with InlC indicates that InlC actively binds Tuba SH3-6 through the slightly concave surface of its central LRR domain (Polle et al., 2014) (Figure 2). The conformation of InlC is unchanged from that of its apo-structure (Ooi et al., 2006) confirming the remarkable rigidity of the LRR domain as similarly observed for the structurally related proteins InlA (Schubert et al., 2002) and InlB (Niemann et al., 2007). The interaction surface of InlC of $\sim 520$ $\AA$ is created by the seven-stranded, parallel $\beta$-sheet of the LRR and is centered around the aromatic residue phenylalanine 146 in LRR4 but involves interactions from each of the LRR. Comparing three symmetrically independent InlC/Tuba SH3-6 complexes that constitute the asymmetric unit of the crystal unit cell indicate that variability of InlC is largely confined to side-chain conformations.

The three-dimensional structure of Tuba SH3-6 is also largely conserved in its interaction with InlC, implying that binding by InlC does not induce major conformational changes in Tuba SH36. The InlC-binding surface of Tuba SH3-6 (570 $\left.\AA^{2}\right)$ involves the $\mathrm{n}$-Src loop, the N-terminal residues of the RT-Src loop, the $3_{10}$-helix and $\beta$-strand 5 . This binding interface substantially overlaps but is not identical to that of the peptide binding groove. However, residues one to eight of the 12-reside peptide substantially clash with InlC in a superposition such that peptide binding to Tuba SH3-6 is eliminated by InlC binding (Polle et al., 2014). 


\section{Displacement of N-WASP by InIC}

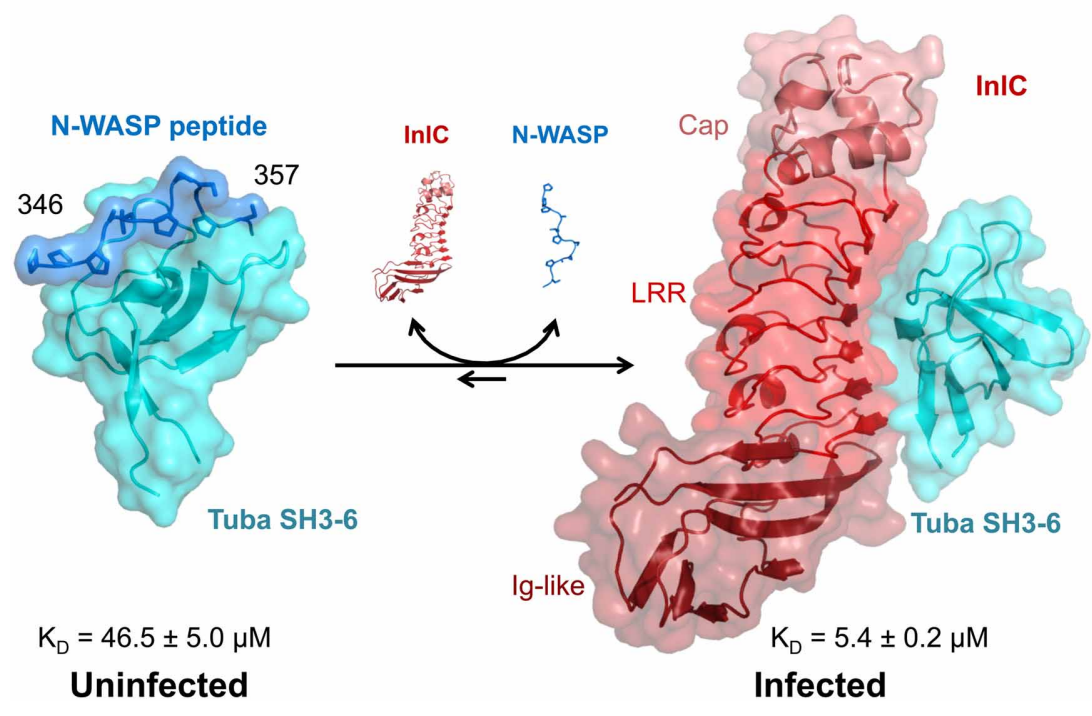

FIGURE 2 | Molecular Displacement of human N-WASP from human Tuba SH3-6 by InIC of Listeria monocytogenes: the critical physiological interaction of N-WASP with Tuba is mediated by the recognition of a proline-rich peptide of N-WASP (blue, residues 346-357) by a peptide binding groove on the sixth $\mathrm{SH} 3$ domain (SH3-6, cyan) of Tuba. This interaction is disrupted by InIC (shades of red: pink-N-terminal Cap domain, red-central leucine rich repeat or LRR domain, maroon-C-terminal Ig-like domain) secreted by L. monocytogenes during infection, which binds to a partly overlapping surface of Tuba SH3-6. While the latter interaction is not itself very tight $\left(K_{D}=5.4 \mu \mathrm{M}\right)$, it is sufficiently tight to outcompete the interaction of N-WASP with Tuba SH3-6 which at $K_{D}=46.5 \mu \mathrm{M}$ is roughly an order of magnitude weaker but typical of transient or reversible, physiological interactions.
Surfaces of InlC and Tuba SH3-6 are roughly complementary such that a significant driving force for complex formation appears to be the entropic exclusion of water molecules. Individual interactions involve $\pi-\pi$ and $\mathrm{CH} \cdot \pi$ stacking, general van der Waals contacts, hydrogen bonds and two imperfect salt bridges. Overall the interaction does not appear to be optimized for tight binding. Correspondingly the dissociation constant was found to be $K_{D}=5.4 \pm 0.2 \mu \mathrm{M}$, indicating a moderately tight interaction. Most importantly the interaction is $\sim 9$ times tighter than that between Tuba SH3-6 and N-WASP, easily out-competing the latter. The physiological relevance of the structurally visualized InlC/Tuba SH3-6 interaction was demonstrated by replacing F146 of InlC by alanine, to prevent its interaction with asparagine 1569 of Tuba SH3-6. In vitro this substitution alone essentially abrogates binding (affinity reduced 90 -fold to $K_{D}=487 \pm 298 \mu \mathrm{M}$ ), while in vivo an engineered Listeria strain carrying this mutation behaves identically to an inlC deletion mutant both in spread of bacteria and in protrusion formation (Polle et al., 2014).

Unexpectedly K173 is not involved in the direct interaction of InlC with the Tuba SH3-6 domain (Polle et al., 2014). Correspondingly in vitro analysis of variant $\mathrm{InlC}^{\mathrm{K} 173 \mathrm{~A}}$ by isothermal titration calorimetry did not indicate altered affinity toward Tuba SH3-6 (Polle et al., 2014). The discrepancy between the in vivo and in vitro importance of this residue remains enigmatic. Resolving this issue may require larger, multi-domain fragments of Tuba being used in the analysis.

\section{OUTSTANDING QUESTIONS AND FUTURE STUDIES}

During the structural studies, the Tuba SH3-6 domain was observed to form dimers, tetramers, and octamers in vitro while crystals show Tuba SH3-6 forming helical arrays of variable lengths involving identical asymmetric monomer-monomer interactions (Polle et al., 2014). It is not clear whether the two oligomerization events are equivalent or whether oligomerization is physiologically relevant for the full-length protein. We are currently identifying point mutations that will prevent oligomerization of this domain in vitro without affecting peptide-ligand binding. In situ production of variant proteins could then indicate whether Tuba SH3-6 oligomerization is physiologically relevant.

Another critical unresolved question is what host physiological processes underlie Tuba-mediated regulation of Listeria spread? Answering this question is challenging, given the multiple functions of Cdc42 and N-WASP in actin assembly, endocytosis, exocytosis, and cell polarity (Harris and Tepass, 2010; Suetsugu and Gautreau, 2012). Here we suggest two possibilities: First, Tuba, N-WASP, and Cdc42 may restrain Listeria protrusions by maintaining actin filaments at cell junctions (Otani et al., 2006; Kovacs et al., 2011). Such filaments act together with myosin II to generate junctional tension (Gomez et al., 2011). Second, Tuba, Cdc42, and N-WASP may indirectly affect cell junctions and Listeria spread by promoting the exocytic delivery of host proteins that generate junctional tension. Interestingly, Tuba, Cdc42, N-WASP each localize to the Golgi apparatus, suggesting a potential role for these proteins in exocytosis (Salazar 
et al., 2003; Matas et al., 2004; Kodani et al., 2009). Future work should elucidate how Tuba, N-WASP, and Cdc42 control Listeria spread. Finally, what are the mechanisms that direct the internalization of Listeria-containing protrusions? A recent study demonstrated that human casein kinase $1-\alpha(C K 1-\alpha)$ is needed for the resolution of protrusions into vacuoles containing Listeria (Chong et al., 2011). Understanding how this kinase promotes protrusion engulfment will likely require the identification of CK1- $\alpha$ substrates involved in bacterial spread. Research in the next decade is anticipated to make considerable progress in understanding how protrusive structures are generated and exchanged between host cells to mediate Listeria spread.

\section{ACKNOWLEDGMENTS}

Work in Keith Ireton's laboratory is supported in part by grants from the National Institutes of Health (R01AI085072) and the Marsden Fund of the Royal Society of New Zealand (UOO1003). Research in Wolf-Dieter Schubert's laboratory is supported by the Medical Research Council of South Africa, the National Research Foundation of South Africa and previously by the Helmholtz Centre for Infection Research, Braunschweig, Germany.

\section{REFERENCES}

Bierne, H., Sabet, C., Personnic, N., and Cossart, P. (2007). Internalins: a complex family of leucine-rich repeat-containing proteins in Listeria monocytogenes. Microbes Infect. 9, 1156-1166. doi: 10.1016/j.micinf.2007. 05.003

Bryant, D. M., Datta, A., Rodríguez-Fraticelli, A. E., Peränen, J., Martín-Belmonte, F., and Mostov, K. E. (2010). A molecular network for de novo generation of the apical surface and lumen. Nat. Cell Biol. 12, 1035-1045. doi: 10.1038/ ncb2106

Carlier, M. F., Nioche, P., Broutin-L'Hermite, I., Boujemaa, R., Le Clainche, C., Egile, C., et al. (2000). GRB2 links signaling to actin assembly by enhancing interaction of neural Wiskott-Aldrich syndrome protein (N-WASp) with actin-related protein (ARP2/3) complex. J. Biol. Chem. 275, 21946-21952. doi: 10.1074/jbc.M000687200

Cemma, M., and Brumell, J. H. (2012). Interactions of pathogenic bacteria with autophagy systems. Curr. Biol. 22, R540-R545. doi: 10.1016/j.cub.2012. 06.001

Chong, R., Squires, R., Swiss, R., and Agaisse, H. (2011). RNAi screen reveals host cell kinases specifically involved in Listeria monocytogenes spread from cell to cell. PLOS ONE 6:e23399. doi: 10.1371/journal.pone.00 23399

Engelbrecht, F., Chun, S. K., Ochs, C., Hess, J., Lottspeich, F., Goebel, W., et al. (1996). A new PrfA-regulated gene of Listeria monocytogenes encoding a small, secreted protein which belongs to the family of internalins. Mol. Microbiol. 21, 823-837. doi: 10.1046/j.1365-2958.1996.541414.x

Gomez, G. A., McLachlan, R. W., and Yap, A. S. (2011). Productive tension: forcesensing and homeostasis of cell-cell junctions. Trends Cell Biol. 21, 499-505. doi: 10.1016/j.tcb.2011.05.006

Gouin, E., Abid-Conquy, M., Balestrino, D., Nahori, M. A., Villiers, V., Colland, F., et al. (2010). The Listeria monocytogenes InlC protein interferes with innate immune responses by targeting the I\{kappa\}B kinase subunit IKK $\{$ alpha\}. Proc. Natl. Acad. Sci. U.S.A. 107, 17333-17339. doi: 10.1073/pnas.10077 65107

Gouin, E., Welch, M. D., and Cossart, P. (2005). Actin-based motility of intracellular pathogens. Curr. Opin. Microbiol. 8, 35-45. doi: 10.1016/j.mib.2004.12.013

Haglund, C. M., and Welch, M. D. (2011). Pathogens and polymers: microbehost interactions illuminate the cytoskeleton. J. Cell Biol. 195, 7-17. doi: $10.1083 /$ jcb. 201103148

Harris, K. P., and Tepass, U. (2010). Cdc42 and vesicle trafficking in polarized cells. Traffic 11, 1272-1279. doi: 10.1111/j.1600-0854.2010.01102.x
Ireton, K. (2013). Molecular mechanisms of cell-cell spread of intracellular bacterial pathogens. Open Biol. 3:130079. doi: 10.1098/rsob. 130079

Jaffe, A. B., and Hall, A. (2005). Rho GTPases: biochemistry and biology. Annu. Rev. Cell Dev. Biol. 21, 247-269. doi: 10.1146/annurev.cellbio.21.020604. 150721

Kodani, A., Kristensen, I., Huang, L., and Sütterlin, C. (2009). GM130dependent control of $\mathrm{Cdc} 42$ activity at the golgi regulates centrosome organization. Mol. Biol. Cell 20, 1192-1200. doi: 10.1091/mbc.E0808-0834

Kovacs, E. M., Makar, R. S., and Gertler, F. B. (2006). Tuba stimulates intracellular N-WASP-dependent actin assembly. J. Cell Sci. 119, 2715-2726. doi: $10.1242 /$ jcs. 03005

Kovacs, E. M., Verma, S., Thomas, S. G., and Yap, A. S. (2011). Tuba and N-WASP function cooperatively to position the central lumen during epithelial cyst morphogenesis. Cell Adh. Migr. 5, 344-350. doi: 10.4161/cam.5.4. 16717

Leung, N., Gianfelice, A., Gray-Owen, S. D., and Ireton, K. (2013). Impact of the Listeria monocytogenes protein InlC on infection in mice. Infect. Immun. 81, 1334-1340. doi: 10.1128/IAI.01377-12

Lim, W. A., Richards, F. M., and Fox, R. O. (1994). Structural determinants of peptide-binding orientation and of sequence specificity in $\mathrm{SH} 3$ domains. Nature 372, 375-379. doi: 10.1038/372375a0

Matas, O. B., Martinez-Menarguez, J. A., and Egea, G. (2004). Association of Cdc42/N-WASP/Arp2/3 signaling pathway with golgi membranes. Traffic 5, 838-846. doi: 10.1111/j.1600-0854.2004.00225.x

Mostowy, S., and Cossart, P. (2012). Bacterial autophagy: restriction or promotion of bacteria replication? Trends Cell Biol. 22, 283-291. doi: 10.1016/j.tcb.2012.03.006

Niemann, H. H., Jäger, V., Butler, P. J., van den Heuvel, J. J., Schmidt, S., Ferraris, D., et al. (2007). Structure of the human receptor tyrosine kinase met in complex with the Listeria invasion protein InlB. Cell 130, 235-246. doi: 10.1016/j.cell.2007.05.037

Ooi, A., Hussain, S., Seyedarabi, A., and Pickersgill, R. W. (2006). Structure of Internalin $\mathrm{C}$ from Listeria monocytogenes. Acta Crystallogr. D Biol. Crystallogr. 62, 1287-1293. doi: 10.1107/S0907444906 026746

Otani, T., Ichii, T., Aono, S., and Takeichi, M. (2006). Cdc42 GEF Tuba regulates the junctional configuration of simple epithelial cells. J. Cell Biol. 175, 135-146. doi: $10.1083 /$ jcb.200605012

Polle, L., Rigano, L. A., Julian, R., Ireton, K., and Schubert, W. D. (2014). Structural details of human Tuba recruitment by InlC of Listeria monocytogenes elucidate bacterial cell-cell spreading. Structure 22, 304-314. doi: 10.1016/j.str.2013. 10.017

Qin, Y., Meisen, W. H., Hao, Y., and Macara, I. G. (2010). Tuba, a Cdc42 GEF, is required for polarized spindle orientation during epithelial cyst formation. J. Cell Biol. 189, 661-669. doi: 10.1083/jcb.201 002097

Rajabian, T., Gavicherla, B., Heisig, M., Müller-Altrock, S., Goebel, W., Gray-Owen, S. D., et al. (2009). The bacterial virulence factor InlC perturbs apical cell junctions and promotes cell-to-cell spread of Listeria. Nat. Cell Biol. 11, 1212-1218. doi: $10.1038 /$ ncb1964

Rigano, L. A., Dowd, G. C., Wang, Y., and Ireton, K. (2014). Listeria monocytogenes antagonizes the human GTPase Cdc42 to promote bacterial spread. Cell. Microbiol. doi: 10.1111/cmi.12260. [Epub ahead of print].

Salazar, M. A., Kwiatkowski, A. V., Pelligrini, L., Cestra, G., Butler, M. H., Rosmann, K. L., et al. (2003). Tuba, a novel protein containing bin/amphiphysin/Rvs and $\mathrm{Dbl}$ homology domains, links dynamin to regulation of the actin cytoskeleton. J. Biol. Chem. 278, 49031-49043. doi: 10.1074/jbc.M3081 04200

Sato, M., Kitaguchi, T., Numano, R., Ikematsu, K., Kakeyama, M., Murata, M., et al. (2012). The small GTPase Cdc42 modulates the number of exocytosis-competent dense-core vesicles in PC12 cells. Biochem. Biophys. Res. Commun. 420, 417-421. doi: 10.1016/j.bbrc.2012. 03.010

Schubert, W. D., Göbel, G., Diepholz, M., Darji, A., Kloer, D., Hain, T., et al. (2001). Internalins from the human pathogen Listeria monocytogenes combine three distinct folds into a contiguous internalin domain. J. Mol. Biol. 312, 783-794. doi: 10.1006/jmbi.2001.4989 
Schubert, W. D., Urbanke, C., Ziehm, T., Beier, V., Machner, M. P., Domann, E., et al. (2002). Structure of Internalin, a major invasion protein of Listeria monocytogenes, in complex with its human receptor E-cadherin. Cell 111, 825-836. doi: 10.1016/S0092-8674(02)01136-4

Suetsugu, S., and Gautreau, A. (2012). Synergistic BAR-NPF interactions in actin-driven membrane remodeling. Trends Cell Biol. 22, 141-150. doi: 10.1016/j.tcb.2012.01.001

Wallace, S. W., Durgan, J., Jin, D., and Hall, A. (2010). Cdc42 regulates apical junction formation in human bronchial epithelial cells through PAK4 and Par6B. Mol. Biol. Cell 21, 2996-3006. doi: 10.1091/mbc.E10-05-0429

Conflict of Interest Statement: The authors declare that the research was conducted in the absence of any commercial or financial relationships that could be construed as a potential conflict of interest.
Received: 20 November 2013; accepted: 05 February 2014; published online: 21 February 2014.

Citation: Ireton K, Rigano LA, Polle L and Schubert W-D (2014) Molecular mechanism of protrusion formation during cell-to-cell spread of Listeria. Front. Cell. Infect. Microbiol. 4:21. doi: 10.3389/fcimb.2014.00021

This article was submitted to the journal Frontiers in Cellular and Infection Microbiology.

Copyright (C) 2014 Ireton, Rigano, Polle and Schubert. This is an open-access article distributed under the terms of the Creative Commons Attribution License (CC BY). The use, distribution or reproduction in other forums is permitted, provided the original author(s) or licensor are credited and that the original publication in this journal is cited, in accordance with accepted academic practice. No use, distribution or reproduction is permitted which does not comply with these terms. 